\title{
A case of cyclist's nodule in a female patient
} \author{
F E Suleman, ${ }^{1} \mathrm{MB} \mathrm{ChB}, \mathrm{MMed}$ Rad (Diag), FCRad (Diag) SA \\ ${ }^{1}$ Department of Radiology, Steve Biko Academic Hospital, Pretoria, South Africa \\ ${ }^{2}$ Department of Radiology, Little Company of Mary, Pretoria, South Africa
}

N Z Makhanya, ${ }^{1}$ MB ChB, MMed Rad (Diag), FCRad (Diag) SA; M Velleman, ${ }^{2}$ MB ChB, MMed Rad (Diag), FC Rad (Diag);

Corresponding author: N Z Makhanya (makhanya.nz@gmail.com)

There are few reported cases of cyclist's nodule in females. The condition has thus lent itself to synonyms such as third, supernumerary or accessory testicle. We report the imaging findings of a perineal nodule in a 29 -year-old female patient who is a known cyclist, and discuss the differential diagnosis.

S Afr J SM 2014;26(3):93-94. DOI:10.7196/SAJSM.561

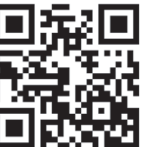

A cyclist's nodule is an infrequently diagnosed yet fairly common condition of the perineum, affecting cyclists. It therefore requires a high index of suspicion in patients who are known cyclists. It is typically a tender, firm, soft-tissue nodule, seldom exceeding $3 \mathrm{~cm}$, located in the paramedian region of the perineum. ${ }^{[1]}$ The overlying skin is usually normal, but may show features of chafing or chronic irritation.

The condition is predominantly described in males, ${ }^{[2]}$ leading to it being termed a third, supernumerary or accessory testicle. Other synonyms include perineal hygroma and perineal induration. ${ }^{[1]}$

\section{Case report}

A 29-year-old female patient, a known cyclist, was referred for imaging following a complaint of a painful, palpable mass on the right inferomedial buttock region.

Ultrasound (US) examination showed a well-circumscribed, heterogenously hypoechoic, solid nodule in the subcutaneous tissues, inferior to the ischial tuberosity, adjacent to the common hamstring origin (Fig. 1). The nodule measured $12 \mathrm{~mm} \times 7 \mathrm{~mm} \times 15 \mathrm{~mm}$ and showed no flow on Doppler.

A magnetic resonance image (MRI) of the pelvis was also performed and showed a poorly circumscribed nodule, isointense to muscle on all sequences, in the right perineum (Fig. 2).

A diagnosis of a cyclist's nodule was made based on the clinical history, the typical location of the lesion and the radiological findings.

The patient was managed conservatively with analgesia and advised to give up cycling. She was reluctant to give up the sport and opted to change the saddle, which, on follow-up, appears to have helped.

\section{Discussion}

A cyclist's nodule is believed to be as a result of repeated microtrauma from vibration and friction between the ischial tuberosities and the saddle, with constant rubbing of the superficial perineal fascia against the bony structures. ${ }^{[1]}$ This induces a combination of fat necrosis,

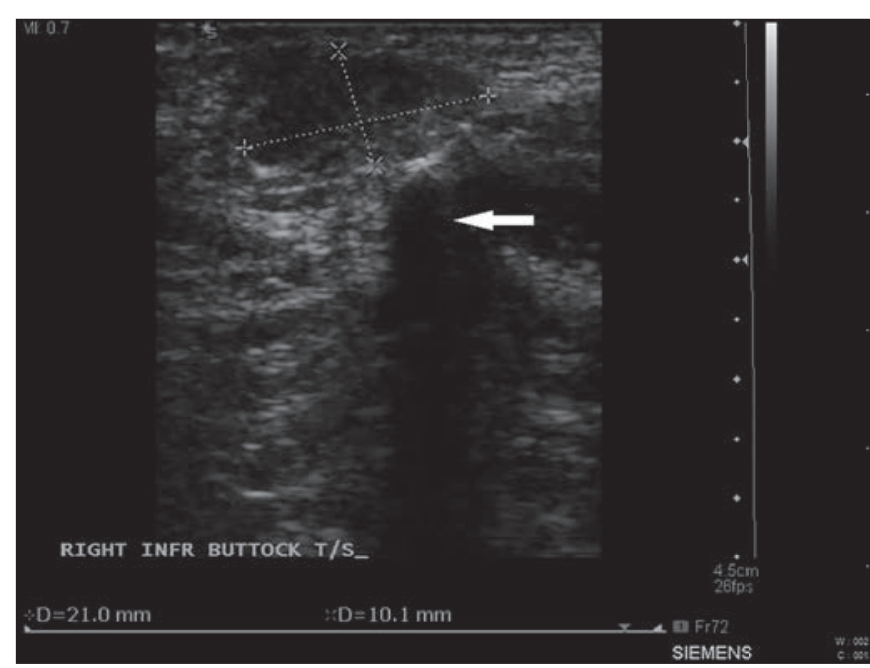

Fig. 1. Ultrasound showing a well-circumscribed hypoechoic oval lesion (measured). Note the posterior acoustic shadow cast by the adjacent ischeal tuberosity (arrow).

collagen degeneration, myxoid changes and sometimes pseudocyst formation. ${ }^{[3]}$ Although histologically few blood vessels are seen, the lesion is not well vascularised.

The nodules are usually fixed to the underlying deep soft tissues and are covered by normal skin. ${ }^{[1]}$ The absence of clinical features of infection excludes the diagnosis of an abscess, a common condition in this region. Rarely, in females, the vulva may demonstrate unilateral lymphoedema of the labia majora, which is attributed to the combination of chronic inflammation and damaged lymphatic flow to the vulvoperineal and inguinal lymphatic vessels. ${ }^{[3]}$

Symptoms include pain on pressure and when sitting on the saddle, which may even require the cyclist to give up the sport. Conservative management such as local corticosteroid injection is described; however, definitive treatment involves removing the causative agent. The lesions have been reported to recur, even after excision. 

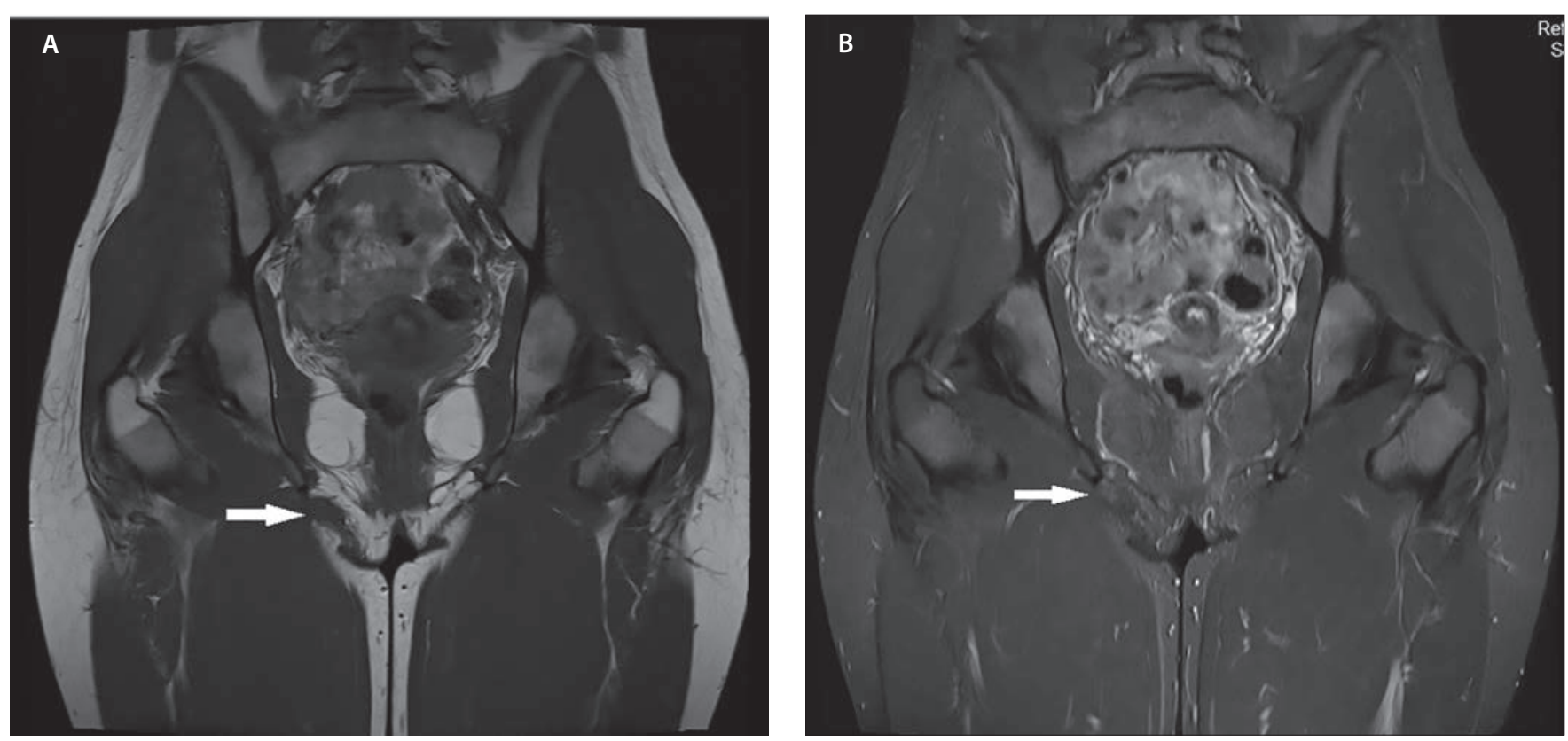

Fig. 2. (A) T1 coronal MRI demonstrating the cyclist's nodule (arrow) as a homogenous lesion, in the right perineal region, which is isointense to muscle. (B) The fluid-sensitive sequence coronal MRI shows the cyclists' nodule (arrow) as a poorly defined nodule, isointense to the overlying subcutaneous fat. No oedema is noted surrounding the nodule. (MRI = magnetic resonance image.)

Most articles report the histological characteristics of the lesions more than the imaging characteristics. If the diagnosis is suspected, then the imaging modality of choice is US, where the diagnosis can be made with certainty. US demonstrates a hypoechoic solid nodule, without any increased Doppler signal. Small internal cystic and/or fatty areas may also be seen, giving the nodule a slightly heterogenous appearance, as in our patient. On computed tomography and MRI, this hypovascular lesion should show no uptake of contrast agent.

The primary role of imaging is to determine the exact extent of the lesion, and in most cases, US will suffice for imaging evaluation. Imaging has an additional role in the differentiation between cyclist's nodule and other causes of perineal swelling. The differential diagnosis includes abscess, epidermal cyst, and benign and malignant tumours (fibroma, soft-tissue sarcoma or metastasis). ${ }^{[3]}$

The MRI was performed at no cost to the patient, for academic purposes of demonstrating the MRI features of a known cyclist nodule. For this reason, no contrast was given. A fluid-sensitive sequence was performed in order to evaluate for features of acute inflammation.
The sonographic findings were more obvious than the MRI findings, where the lesion, being isointense to adjacent soft tissues on all sequences, was fairly difficult to locate.

\section{Conclusion}

Recreational history of cycling should be sought in all patients, including females, when a non-infective soft-tissue nodule in the perineal region is seen, as the diagnosis could be a cyclist's nodule.

\section{References}

1. Van de Perre S, Vanhoenacker FM, Vanstraelen L, Gaens J, Michiels M. Perineal nodular swelling in a recreational cyclist: diagnosis and discussion. Skeletal Radiol 2009;38:933-934. [http://dx.doi.org/10.1007/s00256-009-0731-9]

2. McCluggage WG, Smith JH. Reactive fibroblastic and myofibroblastic proliferation of the vulva (cyclist's nodule): A hitherto poorly described vulval lesion occurring in cyclists. Am J Surg Pathol 2011;35:110-114. [http://dx.doi.org/10.1097/ PAS.0b013e3181ffd8ab]

3. Leibovitch I, Mor Y. The Vicious Cycling: Bicycling Related Urogenital Disorders. Eur Urol 2005;47(3):277-286. [http://dx.doi.org/10.1016/j.eururo.2004.10.024] 